\title{
Saúde auditiva e emissões otoacústicas evocadas em motoristas de uma cidade do interior do Paraná, Brasil
}

Hearing health and evoked otoacoustic emissions in drivers from a town in the state of Paraná, Brazil Salud auditiva y emisiones otoacústicas evocadas en conductores de una ciudad del interior de Paraná, Brasil Carla Rafaela Correia SOARES Juliana De CONTO

Dayane Domeneghini DIDONÉ Mariane AMARAL

Departamento de Fonoaudiologia, Universidade Estadual do Centro Oeste, Unicentro, Campus Irati, 84505-677 Irati-PR, Brasil

\section{Resumo}

Introdução: O ruído é um tipo de som que provoca sensação auditiva desagradável, podendo provocar efeitos auditivos e extra auditivos. Motoristas são profissionais que estão sujeitos a prejuízos de saúde geral e auditiva, pois precisam enfrentar o ruído diariamente em suas atividades profissionais. Objetivo: Verificar a presença ou ausência das emissões otoacústicas evocadas por produto de distorção, queixas auditivas e extra-auditivas, bem como promover uma ação educativa sobre saúde auditiva em um grupo de motoristas de uma cidade do interior do Paraná, Brasil. Métodos: Participaram 21 motoristas e o estudo foi dividido em três etapas, sendo na primeira, a aplicação de questionário, na segunda, inspeção visual do conduto auditivo externo e exame de emissões otoacústicas e na terceira etapa foi realizada ação educativa aos motoristas sobre cuidados em saúde auditiva. Resultados: O sintoma auditivo mais relatado foi a sensação de plenitude auricular e o sintoma extra-auditivo, foi a cefaleia. Quanto às emissões otoacústicas, foi observado que estavam presentes em mais de $70 \%$ das orelhas. Conclusão: Este grupo de motoristas apresenta poucas queixas relacionadas à saúde geral e à audição. No que se refere às emissões otoacústicas, foi observado que estavam presentes na maior parte das orelhas estudadas, exceto nas frequências mais agudas, de 4 e $5 \mathrm{KHz}$. Quanto à ação educativa realizada para esses profissionais, pode-se perceber que os mesmos apresentam poucos conhecimentos sobre os cuidados em saúde auditiva e higienização da orelha, sendo a orientação uma atividade imprescindível para essa população.

Descritores: Saúde do Trabalhador; Ruído; Perda Auditiva.

\section{Abstract}

Introduction: Noise is a type of sound that causes unpleasant auditory sensation, which can cause auditory and extra auditory effects. Drivers are professionals who are subject to general and hearing health impairments, as they need to face noise daily in their professional activities. Objective: To verify the presence or absence of distortion product evoked otoacoustic emissions, auditory complaints, as well as promote an educational action on hearing health in a group of drivers from a town in the state of Paraná, Brazil. Methods: The participants were 21 drivers and the study was divided into three stages, the first being the application of a questionnaire, the second was a visual inspection of the external auditory canal and examination of evoked otoacoustic emissions, and at the third stage, an educational action was performed to drivers on hearing health care. Results: The most reported auditory symptom was the sensation of ear fullness and the extra auditory symptom, was headache. As for otoacoustic emissions, it was observed that they were present in more than $70 \%$ of the ears. Conclusion: This group of drivers presents few complaints related to health and hearing. Regarding otoacoustic emissions, it was observed that most of the ears studied were present, except at high frequencies of 4 and $5 \mathrm{KHz}$. Regarding the educational action performed for these professionals, it can be perceived that they have little knowledge about hearing health care and ear hygiene, and guidance is an indispensable activity for this population.

Descriptors: Occupational Health; Noise; Hearing Loss.

\section{Resumen}

Introducción: El ruido es un tipo de sonido que causa una sensación auditiva desagradable, que puede causar efectos auditivos y extra auditivos. Los conductores son profesionales que están sujetos a daños a la salud y la audición en general, ya que necesitan enfrentar el ruido diariamente en sus actividades profesionales. Meta: Verificar la presencia o ausencia de emisiones otoacústicas provocadas por productos de distorsión, quejas auditivas y extra auditivas, así como promover una acción educativa sobre la salud auditiva en un grupo de conductores de una ciudad en el interior de Paraná, Brasil. Metodología: participaron 21 conductores y el estudio se dividió en tres etapas, en la primera, la aplicación de un cuestionario, en la segunda, inspección visual del canal auditivo externo y examen de las emisiones otoacústicas evocadas, y en la tercera etapa, se llevó a cabo una acción educativa para los conductores sobre la atención en salud auditiva Resultados: El síntoma auditivo más reportado fue la sensación de llenura del oído y el síntoma auditivo adicional fue dolor de cabeza. En cuanto a las emisiones otoacústicas, se observó que estaban presentes en más del $70 \%$ de los oídos. Conclusión: Este grupo de conductores tiene pocas quejas relacionadas con la salud general y la audición. Con respecto a las emisiones otoacústicas, se observó que estaban presentes en la mayoría de los oídos estudiados, excepto en las frecuencias más altas, de 4 y $5 \mathrm{KHz}$. En cuanto a la acción educativa llevada a cabo para estos profesionales, se puede ver que tienen poco conocimiento sobre el cuidado de la salud auditiva y la higiene del oído, y la orientación es una actividad esencial para esta población.

Descriptores: Salud Laboral; Ruido; Pérdida Auditiva.

INTRODUÇÃO

O ruído é um tipo de som que provoca sensação auditiva desagradável podendo interferir na percepção de outros sons, principalmente os da fala ${ }^{1}$, além de interferir em atividades cotidianas básicas como dormir, descansar, estudar e se comunicar, podendo causar doenças do coração, problemas de saúde mental e dano auditivo ${ }^{2}$. O incômodo causado pelo ruído pode levar a diversas respostas negativas como a ansiedade, agitação, decepção, depressão, distração, exaustão, insatisfação e raiva ${ }^{2}$.

Além disso, a exposição sistemática ao ruído pode provocar efeitos auditivos, tais como o zumbido e o incômodo a sons intensos e ainda afetar o funcionamento das células ciliadas da cóclea $^{3}$. Para a verificação de presença desses e de outros sintomas auditivos e extra auditivos em populações expostas de forma sistemática ao ruído, é possível utilizar questionários direcionados para o tema. 
De acordo com a Norma Regulamentadora $\mathrm{n}^{\circ} 15$ do Ministério do Trabalho, a exposição ao ruído afeta primeiro as frequências de 3, 4 e $6 \mathrm{KHz}$, contudo tal efeito depende da intensidade e duração do ruído, bem como da predisposição às alterações auditivas de cada indivíduo ${ }^{4}$. Visto que tal exposição pode causar danos na cóclea, é possível utilizar 0 exame de Emissões Otoacústicas Evocadas por Produto de Distorção (EOE-PD) para avaliar a funcionalidade das células ciliadas da cóclea de indivíduos expostos ao ruído, pois trata-se de uma técnica não invasiva, rápida e objetiva. As EOE-PD asseguram um diferencial no diagnóstico de alterações auditivas relacionadas ao ruído por se tratar de um teste prático que avalia frequências específicas de 2, 3, 4 e 5 $\mathrm{KHz}$, podendo verificar a função coclear de forma segura e são sensíveis aos estágios iniciais de alterações na coclea ${ }^{5}$.

Motoristas são profissionais que estão sujeitos a tais prejuízos de saúde geral e de saúde auditiva, pois precisam enfrentar o ruído diariamente, pois fazem parte de um grupo de profissionais que estão expostos a diversos riscos durante a jornada de trabalho, como as condições de estradas, o ruído do motor do veículo e das rodovias que apresentam diversos automóveis ruidosos ${ }^{6}$. Ruídos intensos e permanentes podem causar vários distúrbios, alterando significativamente a capacidade de concentração, fator relevante na profissão do motorista $^{7}$.

Quanto maior for o cuidado e atenção desses profissionais em suas atividades laborativas, maior será a sua segurança e a do próximo, pois o ruído é responsável pela poluição sonora do trânsito, sendo nocivo para a saúde auditiva humana ${ }^{8}$.

Diante do exposto, o objetivo da presente pesquisa é de verificar a presença ou ausência das EOE-PD, as queixas auditivas e extra auditivas, bem como promover uma ação educativa sobre saúde auditiva em um grupo de motoristas de uma cidade do interior do Paraná, Brasil.

\section{MATERIAL E MÉTODO}

Trata-se de um estudo exploratório, descritivo e de abordagem quantitativa, aprovado pelo Comitê de Ética em Pesquisa da Universidade Estadual do Centro Oeste, Unicentro - Campus Irati (PR), sob parecer $\mathrm{n}^{\circ}$ 3.417.385. Todos os participantes assinaram o Termo de Consentimento Livre e Esclarecido.

A coleta de dados foi realizada na Clínica Escola de Fonoaudiologia da
Universidade Estadual do Centro Oeste, Unicentro - Campus Irati (PR). Foram incluídos apenas sujeitos que trabalham profissionalmente como motoristas e foram excluídos aqueles nos quais havia presença de rolha de cera observada durante inspeção visual do conduto auditivo externo e/ou que não participaram de todas as etapas do estudo.

$\mathrm{Na}$ primeira etapa do estudo os participantes responderam a um questionário adaptado do estudo desenvolvido por Oliveira et $\mathrm{al}^{9}$, a fim de coletar dados para caracterização da amostra, informações referentes a atuação profissional, saúde geral e saúde auditiva. Os participantes responderam ao questionário individualmente e no caso de alguma dificuldade de compreensão das questões, a pesquisadora fez os esclarecimentos necessários a respeito.

$\mathrm{Na}$ segunda etapa do estudo, os participantes passaram por triagem auditiva, sendo realizada inspeção visual do conduto auditivo externo e em seguida foi realizado o exame de EOE-PD em modo triagem, utilizando o aparelho Otoread da marca Interacoustics. As EOE-PD foram consideradas presentes quando registraram relação sinal-ruído superior a seis decibéis em três ou mais frequências pesquisadas $^{10}$, sendo estas as frequências de 2 , 3,4 e $5 \mathrm{KHZ}$. O registro das EOE-PD ocorre pelo registro de sinais sonoros que acontecem após a estimulação de dois tons puros de frequências diferentes, apresentados simultaneamente à cóclea $^{10}$. As frequências primárias selecionadas para a avaliação seguiram a proporção de $f 2 / f 1=1,22$ e a relação de intensidade foi de $L 1=65$ dB NPS e $L 2=$ $55 \mathrm{~dB}$ NPS.

$\mathrm{Na}$ terceira etapa do estudo, imediatamente após a aplicação do questionário e exames, foi realizada uma ação educativa por meio de roda de conversa e orientação aos motoristas, de forma individual ou em grupos, conforme a disponibilidade dos mesmos. Foram abordados temas relacionados à cuidados em saúde auditiva e higienização da orelha, como evitar colocar objetos pontiagudos na orelha para coçar ou mesmo para limpar, pois a cera é uma proteção para a orelha e utilizar somente a toalha úmida após o banho para limpar a parte externa da orelha. Os dados coletados foram armazenados em tabelas do Excel e receberam análise estatística descritiva.

RESULTADOS E DISCUSSÃO

Em relação à caracterização da amostra deste estudo, o grupo foi composto por 21 motoristas, todos do sexo masculino, com faixa etária entre 27 e 56 anos. A predominância do 
sexo masculino nesta categoria profissional é amplamente relatada na literatura ${ }^{2,10-12}$. Em revisão de literatura realizada por Souza et al. ${ }^{13}$, sobre trabalhadores do transporte coletivo urbano no Brasil, os autores identificaram que a prevalência do sexo masculino nessa profissão se dá por conta de uma seleção intencional por parte de proprietários das empresas de veículos, pois conhecem a rotina desgastante que esse tipo de ocupação impõe ao profissional.

Quanto à jornada de trabalho, o predomínio foi de seis a oito horas diárias $(57,14 \%)$ e tipo de veículo mais utilizado pelos motoristas participantes desta pesquisa é a van $(28,5 \%)$.

Com relação às questões ocupacionais, oito motoristas $(38,1 \%)$ referem ter trabalhado anteriormente em ambientes ruidosos, dado semelhante ao encontrado no estudo de Oliveira et al. $^{9}$, no qual oito funcionários $(22,2 \%)$ relataram trabalhar em ambiente ruidoso anteriormente.

Onze motoristas $(52,4 \%)$ relataram que não consideram seu veículo ruidoso, fato que pode estar associado com a não percepção do efeito prejudicial do ruído, necessitando investigar de forma mais aprofundada a questão e a partir disso desenvolver ações de conscientização e proteção auditiva.

Além disso, 15 motoristas (66,6\%) referem que mantém o rádio ligado durante 0 trabalho. No estudo de Grudtner e Fiorini², o rádio foi citado por $69,9 \%$ dos participantes como uma das principais fontes de ruído no interior do veículo. Para Oliveira et al. ${ }^{9}$, nos centros urbanos, as principais fontes de ruído são os veículos automotores e de acordo com Lopes et al. ${ }^{11}$, o ruído ocupacional pode ocasionar diversos problemas para saúde, contribuindo assim, para acidentes de trabalho

Outros detalhes da caracterização da amostra podem ser vistos na Tabela 1 .

No que se refere aos sintomas auditivos, $95,2 \%$ dos participantes não perceberam alterações auditivas, $47,6 \%$ referem que sua audição é boa e $76,2 \%$ afirmam não perceber diferenças na percepção auditiva entre as duas orelhas.

Com relação à otalgia, três motoristas $(14,3 \%)$ referem o sintoma. Segundo Tonello ${ }^{14}$, o sintoma otalgia é mencionado com pouca frequência pela literatura por esses profissionais e em estudo que a autora realizou com motoristas de ônibus, apenas 13,8\% dos pacientes com audiometria sugestiva de perda auditiva induzida por ruído (PAIR) relataram o sintoma.
Tabela 1. Caracterização da amostra de motoristas.

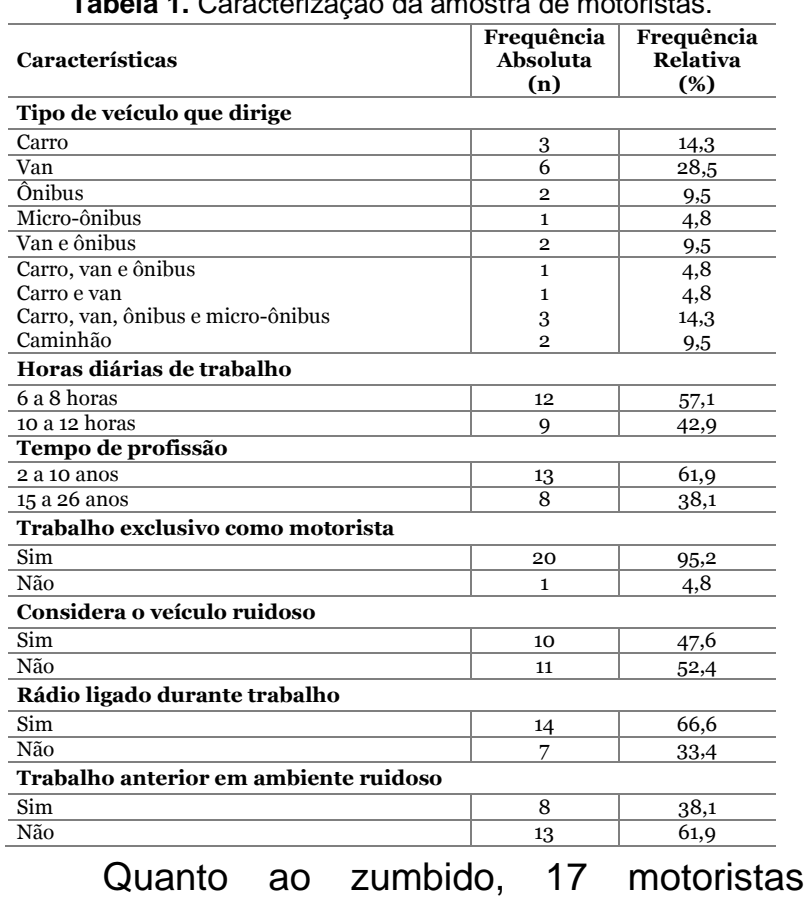

$(80,9 \%)$ afirmaram não apresentar este sintoma. No estudo realizado por Lopes et al ${ }^{11}$, pode-se perceber dado semelhante, pois apenas um participante $(1,32 \%)$ referiu ter zumbido e os demais negam essa queixa. Segundo Lacerda et al. ${ }^{6}$, muitas vezes o ruído ocupacional é 0 agente responsável por ocasionar o zumbido em trabalhadores. De acordo com Bertuol et al. ${ }^{15}$, o zumbido é uma sensação auditiva que ocorre quando não há estimulo sonoro no meio externo. Para Steinmet et al. ${ }^{16}$, o zumbido é um sintoma auditivo amplamente relatado por sujeitos expostos ao ruído ocupacional. Dessa forma, o dado encontrado na presente pesquisa difere da literatura sobre o tema possivelmente pelo tempo de exposição ao ruído e pela suscetibilidade individual.

No que se refere à plenitude auricular 15 motoristas (71,4\%) não apresentaram esse sintoma, enquanto 16 participantes (76,19\%) referiram não apresentar intolerância a sons intensos como mostra na Tabela 2.

$$
\text { Com relação aos sintomas }
$$

extra-auditivos, dois participantes $(9,5 \%)$ apresentaram tontura e seis $(28,6 \%)$ citaram ter cefaleia, dados que se diferenciam da pesquisa realizada por Lacerda et al. ${ }^{6}$, na qual $40 \%$ dos participantes relataram ter dores de cabeça.

Três motoristas (14,2\%) relataram apresentar dificuldades para conversar em ambientes ruidosos, dado que difere do estudo realizado por Oliveira et al. $^{9}$, no qual $33,3 \%$ referiram dificuldades para conversar em ambientes ruidosos.

Três motoristas (14,2\%) referiram ter irritabilidade, dado semelhante ao encontrado 
no estudo de Batista et al. ${ }^{17}$, no qual $15,6 \%$ dos participantes relataram o sintoma. Além disso, quatro $(19,1 \%)$ apresentaram dificuldades para compreender palavras, quatro $(19,1 \%)$ apresentaram alteração no sono, dados que também se diferenciam do estudo de Oliveira et al. ${ }^{9}$, visto que $33,3 \%$ relataram alteração no sono.

Tabela 2. Saúde Geral e Auditiva dos motoristas

\begin{tabular}{|c|c|c|}
\hline & $\begin{array}{l}\text { Frequência } \\
\text { Absoluta } \\
\text { (N) }\end{array}$ & $\begin{array}{c}\text { Frequência } \\
\text { Relativa } \\
\text { (\%) }\end{array}$ \\
\hline \multicolumn{3}{|l|}{ Alterações auditivas declaradas } \\
\hline Sim & 1 & 4.8 \\
\hline Não & 20 & 95,2 \\
\hline \multicolumn{3}{|l|}{ Como considera sua audição } \\
\hline Excelente & 4 & 19,1 \\
\hline Muito boa & 6 & 28,5 \\
\hline Boa & 10 & 47,6 \\
\hline Razoável & 1 & 4,8 \\
\hline \multicolumn{3}{|l|}{ Percepção de uma orelha melhor que a outra } \\
\hline Sim & 5 & 23,8 \\
\hline Não & 16 & 76,2 \\
\hline Orelha Direita melhor & 4 & 80 \\
\hline Orelha Esquerda melhor & 1 & 20 \\
\hline \multicolumn{3}{|l|}{ Exame audiológico anterior } \\
\hline Sim & 15 & 71,4 \\
\hline Não & 6 & 28,6 \\
\hline $\begin{array}{l}\text { Declara resultado dentro dos padrões de } \\
\text { normalidade }\end{array}$ & 14 & 93,4 \\
\hline Declara perda auditiva & 1 & 6,6 \\
\hline \multicolumn{3}{|l|}{ Otalgia } \\
\hline Sim & 3 & 14,3 \\
\hline Não & 18 & 85,7 \\
\hline \multicolumn{3}{|l|}{ Vazão de secreção } \\
\hline Sim & 1 & 4,8 \\
\hline Não & 20 & 95,2 \\
\hline \multicolumn{3}{|l|}{ Uso de medicação contínua } \\
\hline Sim & 3 & 14,3 \\
\hline Não & 18 & 85,7 \\
\hline \multicolumn{3}{|l|}{ Cirurgia de orelha } \\
\hline Sim & o & 0 \\
\hline Não & 21 & 100 \\
\hline \multicolumn{3}{|l|}{ Antecedente familiar de alteração auditiva } \\
\hline Sim & 4 & 19,1 \\
\hline Não & 16 & 76,1 \\
\hline Não sabe referir & 1 & 4,8 \\
\hline \multicolumn{3}{|l|}{ Zumbido } \\
\hline Sim & 4 & 19,1 \\
\hline Não & 17 & 80,9 \\
\hline Zumbido de intensidade baixa & 2 & 50 \\
\hline Zumbido de intensidade média & 2 & 50 \\
\hline Plenitude auricular & & \\
\hline Sim & 6 & 28,6 \\
\hline Não & 15 & 71,4 \\
\hline Sensação de intensidade baixa & 4 & 66,7 \\
\hline Sensação de intensidade média & 2 & 33,4 \\
\hline Tontura & & \\
\hline Sim & 2 & 9,5 \\
\hline Não & 19 & 90,4 \\
\hline Intolerância a sons intensos & & \\
\hline Sim & 5 & 23,8 \\
\hline Não & 16 & 76,2 \\
\hline Cefaleia & & \\
\hline Sim & 6 & 28,6 \\
\hline Não & 15 & 71,4 \\
\hline Irritabilidade & & \\
\hline Sim & 3 & 14,3 \\
\hline Não & 18 & 85,7 \\
\hline Dificuldade para compreender palavras & & \\
\hline Sim & 4 & 19,1 \\
\hline Não & 17 & 80,9 \\
\hline Desatenção & & \\
\hline Sim & 1 & 4,8 \\
\hline Não & 20 & 95,2 \\
\hline Dificuldade de conversação em ambient & uidosos & \\
\hline Sim & 3 & 14,2 \\
\hline Não & 18 & 85,7 \\
\hline Alterações de sono & & \\
\hline Sim & 4 & 19,1 \\
\hline Não & $\frac{4}{17}$ & 80,9 \\
\hline Mal estar após o trabalho & & \\
\hline Sim & 2 & 9,5 \\
\hline Não & 19 & 90,4 \\
\hline Prática de atividade ruidosa após o trab & & \\
\hline Sim & 1 & 4,8 \\
\hline Não & 20 & 95,2 \\
\hline
\end{tabular}

A análise das EOE-PD pode ser vista detalhadamente na Tabela 3. Foi observado que em mais de $70 \%$ das orelhas, as EOE-PD estavam presentes na triagem, de forma que na frequência de $2 \mathrm{KHz}$ estavam presentes em 38 $(90,48 \%)$ das 42 orelhas estudadas. $\mathrm{Na}$ frequência de $3 \mathrm{KHz}$ houve presença em 35 orelhas (83,34\%), na frequência de $4 \mathrm{KHz}$ houve 26 orelhas $(61,91 \%)$ com EOE-PD presentes e na frequência de $5 \mathrm{KHz}$, estavam presentes em 25 orelhas $(59,53 \%)$.

Tabela 3. EOE-PD por frequência em 42 orelhas estudadas de 21 motoristas

\begin{tabular}{|l|l|l|l|l|l|c|c|c|}
\hline Frequência & \multicolumn{2}{|c|}{$\mathbf{2 K H z}$} & \multicolumn{2}{|c|}{$\mathbf{3} \mathbf{K H z}$} & \multicolumn{2}{|c|}{$\mathbf{4} \mathbf{~ K H z}$} & \multicolumn{2}{|c|}{$\mathbf{5} \mathbf{~ K H z}$} \\
\hline & $\mathbf{N}$ & $\mathbf{\%}$ & $\mathbf{N}$ & $\mathbf{\%}$ & $\mathbf{n}$ & $\mathbf{\%}$ & $\mathbf{n}$ & $\mathbf{\%}$ \\
\hline Presentes & 38 & 90,48 & 35 & 83,34 & 26 & 61,91 & 25 & 59,53 \\
\hline Ausentes & 4 & 9,52 & 7 & 16,66 & 16 & 38,09 & 17 & 40,47 \\
\hline Total & 42 & 100 & 42 & 100 & 42 & 100 & 42 & 100 \\
\hline
\end{tabular}

Contudo, no que se refere às EOE-PD ausentes, estas foram em sua maioria nas frequências mais agudas, de 4 e $5 \mathrm{KHz}$, sendo que na frequência de $4 \mathrm{KHz}, 16$ orelhas (38,09\%) apresentaram EOE-PD ausentes e em $5 \mathrm{KHz}, 17$ orelhas $(40,47 \%)$ apresentaram ausência de resposta.

De acordo com Fiorini et al. ${ }^{18}$, a ausência nas frequências mais agudas evidencia a instalação de PAIR. Segundo Morata e Lemasters $^{19}$, a PAIR se desenvolve gradativamente, é caracterizada como neurossensorial e irreversível, desenvolvida em um período de seis anos que se inicia nas frequências altas como $6,4,8,3,2 \mathrm{KHz}$ ou 4, 6, $8,3,2 \mathrm{KHz}$. O período citado de seis anos para o início do acometimento auditivo nas frequências citadas acima, pode justificar a presença de EOE-PD na maioria dos motoristas do presente estudo, visto que muitos apresentam menos de dez anos na profissão.

De acordo com Biron ${ }^{20}$, as emissões otoacústicas são consideradas importantes na monitorização da audição de trabalhadores expostos ao ruído, pois os danos causados pela exposição são detectáveis nas emissões, mesmo antes de serem identificados ou visíveis no audiograma.

No estudo de Costa et al. ${ }^{21}$, com trabalhadores expostos ao ruído, as falhas encontradas nas emissões otoacústicas se concentraram nas frequências de 3, 4, 6 e 8 $\mathrm{KHz}$.

Na terceira etapa da pesquisa, conforme a disponibilidade de cada participante, foi realizada uma ação educativa com orientações sobre saúde auditiva, com participação ativa desses profissionais. Vale destacar que a participação dos motoristas nas conversas sobre saúde auditiva é fundamental para a conscientização de hábitos que podem ser 
prejudiciais a audição. De acordo com Gonçalves e Guida22, a educação em saúde deve oferecer condições para que as pessoas desenvolvam 0 senso de responsabilidade, tanto pela sua própria saúde como pela saúde da comunidade. Além disso, a promoção de saúde auditiva proporciona uma melhor qualidade de vida e bem-estar para a população ${ }^{23}$.

\section{CONCLUSÃO}

O transporte mais utilizado por esses profissionais é a van. Quanto aos sintomas auditivos o mais relatado foi plenitude auricular $(28,6 \%)$ e quanto aos sintomas extra auditivos, o mais relatado foi cefaleia $(28,6 \%)$, podendo-se concluir que este grupo de motoristas de uma cidade do interior do Paraná possui poucas queixas relacionadas à saúde geral e à audição.

No que se refere as EOE-PD, foi observado que a maior parte das orelhas as EOE-PD estavam presentes na triagem, exceto nas frequências mais agudas, de 4 e $5 \mathrm{KHz}$. Quanto a orientação realizada para esses profissionais, pode-se perceber que os mesmos apresentam pouco conhecimentos sobre os cuidados em saúde auditiva e higienização da orelha, sendo a orientação sobre cuidados com saúde auditiva imprescindível para essa população. Dadas às limitações do presente estudo, sugere-se que para futuras pesquisas, sejam realizados estudos com maior variação de sexo e uma quantidade maior de indivíduos.

\section{REFERÊNCIAS}

1. Teti BS, Cruz FM, Lago EMG, Zlatar T. Perdas auditivas induzidas pelo ruído no ambiente ocupacional da construção civil. REPA. 2019; 4(1):146-53.

2. Grudtner MJS, Fiorini AC. Ruído urbano e efeitos na saúde de taxistas do Município de São Paulo. Rev UNICET. 2018;1(1):1-17.

3. Andrade WTL, LIMA MAR, SOARES JFR. Queixas auditivas de trabalhadores de uma indústria cerâmica da cidade de João Pessoa/PB. Rev CEFAC. 2015;17(6):1874-81.

4. BRASIL. Ministério do Trabalho. Portaria no 3214 de 08 de junho de 1978. Descreve a Norma Regulamentadora $\mathrm{n}^{\circ} 15$ (NR15) Atividades e Operação Insalubres. Diário Oficial da República Federativa do Brasil, Brasilia (DF); 1978 Jun 08; Suplemento.

5. Coelho MSB, Ferraz JRS, Almeida EOC, Filho NA. As emissões otoacústicas no diagnóstico diferencial das perdas auditivas induzidas por ruído. Rev CEFAC. 2010;12(6):1050-58.

6. Lacerda A, Figueiredo G, Massarolo Neto J, Marques JM. Achados audiológicos e queixas relacionadas à audição dos motoristas de ônibus urbano. Rev Soc Bras Fonoaudiol. 2010;15(2):161-66.

7. Giuliani A. O nível de ruído próximo aos motoristas de ônibus urbano na cidade de Porto Alegre, RS. Rev Liberato. 2011;12(17): 98-103.

8. Godinho RD, Mourão RF, Castro LA, Pouzas RS, Rocha LLv. Ruído Ambiental e a Saúde do Motorista. BJSCR. 2016;13(4):96-100.

9. Oliveira RC, Santos JN, Rabelo ATV, Magalhães MC. O impacto do ruído em trabalhadores de Unidades de Suporte Móveis. Rev CODAS. 2015;27(3):215-22.

10. Sousa LCA, Piza MRT, Alvarenga KF, Cóser PL. Eletrofisiologia da audição e emissões otoacústicas: princípios e aplicações clínicas. Ribeirão Preto: Novo Conceito; 2010.

11. Lopes AC, Otowiz VG, Lopes BMP, Lauris JRP, Santos CC. Prevalência de perda auditiva induzida por ruído em motoristas. Rev Int Arch Otorhinolaryngol. 2012;16(4):509-14.

12. Guardiano JS, Chagas TZ, Slomp Junior H. Avaliação da perda auditiva em motoristas de ônibus de Curitiba. Rev CEFAC.2014;16(1): 50-4.

13. Souza LPS, Silva JJ, Silva CSO, Pinto IK. Prevalência e fatores associados à hipertensão em trabalhadores do transporte coletivo urbano no Brasil. Rev bras med trab. 2017;15(1):80-7.

14. Tonello, EF. Estúdio sobre o perfil audiológico em motoristas de ônibus de uma empresa de transporte coletivo na grande Curitiba-PR [ dissertação] Curitiba: Departamento de Saúde Comunitária, Universidade Federal do Paraná (UFPR); 2014.

15. Bertuol B, Scremin ALX, MarquesPM, Ferreira L, Araújo TM, Biaggio EPV. Zumbido, qualidade de vida e questões emocionais de sujeitos usuários de próteses auditivas. Disturb Comun. 2018;30(1):80-9.

16. Steinmetz LG, Zeigelboim BS, Lacerda AB, Morata TC, Marques JM. Características do zumbido em trabalhadores expostos a ruído. Rev Bras Otorrinolaringol.2009;75(1):714.

17. Batista APL, Rodrigues GAA, Aquino KO, Silva WT. Sintomas auditivos e não auditivos em motoristas de transporte rodoviário. Rev Bionorte. 2015;4(1):86-97.

18. Fiorini AC, Moran MESP. Emissões otoacústicas-produto de distorção: estudo de diferentes relações de níveis sonoros no teste em indivíduos com e sem perdas auditivas. Distúrb Comum. 2005;17(3)385-96.

19. Morata TC, Lemasters GK. Considerações epidemiológicas para o estudo de perdas auditivas ocupacionais. In: Nudelmann AA, Costa EA, Seligman J, Ibagñez RN. PAIR: perda auditiva induzida pelo ruído. Revinter. $2001 ;(2): 1-16$. 
20. Biron EJM. A detecção precoce da perda auditiva induzida pelo ruído com o uso das emissões otoacústicas produto de distorção. Curitiba-PR [dissertação]. Curitiba: Departamento de Saúde Comunitária, Universidade Federal do Paraná (UFPR); 2016.

21. Costa JB, Rosa SAB, Borges LL, Camarano $\mathrm{MRH}$. Caracterização do perfil audiológico em trabalhadores expostos a ruídos ocupacionais. EVS. 2015;42(3):273-87.

22. Gonçalves CGO, Guida HL. Saúde Ambiental: Ação em vigilância em saúde do trabalhador exposto a agentes otoagressivos. In: Boéchat EM, Menezes PL, Couto CM, Frizzo ACF, Scharlach RC, Anastasio ART. Tratado de Audiologia. Rio de Janeiro: Editora Guanabara Koogan, 2015.

23. Lacerda ABM, Soares VMN, Goncalves CGO, Lopes FC, Testono R. Oficinas educativas como estratégia de promoção da saúde auditiva do adolescente: estudo exploratório. Rev ACR. 2013;18(2):85-92.

\section{CONFLITO DE INTERESSES}

Os autores declaram não haver conflitos de interesse

\section{AUTOR PARA CORRESPONDÊNCIA}

\section{Mariane Amaral}

Departamento de Fonoaudiologia, Universidade Estadual do Centro Oeste - Unicentro, Campus Irati 84505-677 Irati-PR, Brasil

E-mail:marianeamaral@unicentro.br 